of Internal Medicine, Chaidari, Greece; ${ }^{2}$ Attikon University Hospital, 2nd Department of Dermatology and Venereology, Chaidari, Greece; ${ }^{3}$ National And Kapodistrian University of Athens, Department of Clinical Therapeutics, Athens, Greece; ${ }^{4}$ Attikon University Hospital, Athens, Greece

Background: Psoriatic arthritis (PsA) affects both sexes equally, however there seem to be significant differences in disease expression between the genders. Objectives: To investigate gender differences in disease manifestations, patient-reported outcomes and comorbidities among patients with PsA.

Methods: This cross-sectional study of patients with PsA followed at an academic rheumatology outpatient clinic between 1/6/2017 and 1/12/2019. We compared clinical characteristics, patient-reported outcomes, disease activity and comorbidities in male and female patients with PSA. All patients were over 18 years of age and fulfilled the CASPAR criteria for PSA. Differences between gender in values of continuous variables were assessed by T-tests or Mann-Whitney tests. The association between categorical variables and gender was assessed by Pearson chi-square test or Fisher's exact test. Results: 135 patients, 83 (62\%) women and 52 (38\%) men were included. Factors studied for gender differences are shown in Table 1 . Women had significantly more tender (11 vs 3 p 0.001) and swollen (10 vs 3, p 0.013) joints, worse VAS (Visual Analogue Scale $0-10$ ) pain ( 6 vs $5, p<0.001$ ), higher ESR ( 20 vs $11, p 0.001$ ) and worse DAPSA(Disease Activity in Psoriatic Arthritis) (33 vs 18 p 0.006) and presented with more enthesitis $(32.5 \%$ vs $13.5 \%$, p 0.013$)$. In contrast, men achieved Minimal Disease Activity (MDA) more frequently $(26.9 \%$ vs $3.6 \% p<0.001)$ and had significantly more comorbidities than women. Polyarthritic disease was more frequent in women $(62 \%$ vs $31 \%)$, although at non-significant levels.

Conclusion: Male patients with PsA have more comorbidities, while female

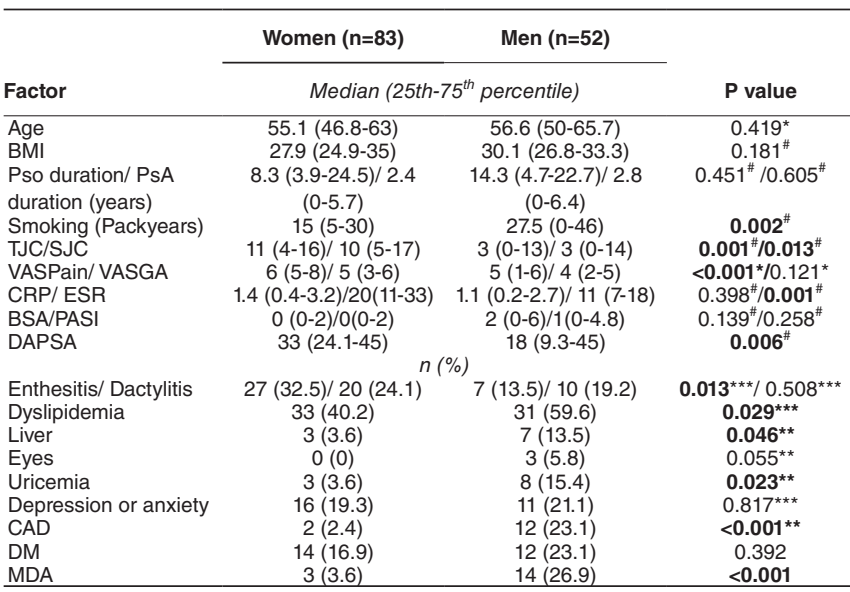

*: T-test with unequal variances; ": Mann-Whitney test; ${ }^{* *}$ : Fisher's exact test; ${ }^{* * *}$ : Pearson chi2 test;

Pso: Psoriasis; PsA: Psoriatic arthritis; BMI: Body mass index; TJC: Tender joint count; SJC: Swollen joint count; VASPain: Visual analogue scale 0-10 for pain; VASGA: Visual analogue scale 0-10 for general assessement; CRP: C-reactive protein; ESR: Erythrocyte sedimentation rate; BSA: Body surface area; PASI: Psoriasis area severity index; DAPSA: Disease activity in psoriatic arthritis; CAD: Coronary artery disease; DM: Diabetes mellitus; MDA: Minimal disease activity;

patients have greater disease activity, worse patient reported outcomes and achieve MDA less frequently.

References:

[1] Determinants of Patient-Reported Psoriatic Arthritis Impact of Disease: An Analysis of the Association with Gender in 458 Patients from 14 Countries.

[2] Orbai AM, Perin J, et al Arthritis Care Res (Hoboken). 2019 Oct 14. doi: 10.1002/acr.24090.

Disclosure of Interests: ALEXANDROS GRIVAS: None declared, IRENE KAPNIARI: None declared, KIMON TZANNIS: None declared, Dimitrios Tseronis: None declared, Michail Aggelakos: None declared, Dimitra Kassara: None declared, KATERINA HAVATZA: None declared, Sofia Flouda: None declared, Dionysis Nikolopoulos: None declared, Theofanis Karageorgas: None declared, EVAGELIA PAPADAVID: None declared, DIMITRIOS BOUMPAS Grant/research support from: Unrestricted grant support from various pharmaceutical companies, PELAGIA KATSIMPRI: None declared DOI: 10.1136/annrheumdis-2020-eular.3346

\section{AB0789 \\ PERSISTENCE IN USE OF BIOLOGIC DISEASE MODIFYING ANTIRHEUMATIC DRUGS TREATMENT IN PATIENTS WITH PSORIATIC ARTHRITIS}

Y. Jin ${ }^{1}$, S. Chen ${ }^{1,2}$, H. Lee ${ }^{1}$, J. Landon ${ }^{1}$, J. F. Merola ${ }^{3}$, S. Kim ${ }^{1,2} .{ }^{1}$ Brigham and Women's Hospital, Division of Pharmacoepidemiology and

Pharmacoeconomics, Boston, United States of America; ${ }^{2}$ Brigham and
Women's Hospital, Division of Rheumatology, Immunology and Allergy, Boston, United States of America; ${ }^{3}$ Brigham and Women's Hospital, Department of Dermatology, Boston, United States of America

Background: Treatment for patients with moderate to severe psoriatic arthritis (PsA) relied on TNF inhibitors (TNFi) for many years. Recent approvals of newe biologics include interleukin (IL) -12 inhibitor ustekinumab and IL-17A inhibitors ixekizumab and secukinumab. Limited up-to-date evidence exists for the comparison of utilization patterns between TNFi and IL inhibitors.

Objectives: To compare the persistence on treatment with biologic disease-modifying anti-rheumatic diseases (DMARDs) in PsA patients who initiated TNFi versus IL inhibitors.

Methods: We conducted a cohort study using a US commercial insurance database (IBM MarketScan: 2014-2017). We identified patients with PsA by using a validated claims-based algorithm (positive predictive value of $82.4 \%$ ) which required two PsA diagnosis codes and a prescription dis pensing for TNFi (etanercept, infliximab, adalimumab, certolizumab, golimumab) or IL inhibitor (secukinumab, ustekinumab, or ixekizumab). The index date was the $1^{\text {st }}$ drug dispensing date after the $2^{\text {nd }}$ PsA diagnosis. We excluded patients with biologic DMARD use at any time prior to the index date. Patients were $\geq 18$ years old on the index date and continuously enrolled in the plan for $\geq 365$ days prior and after the index date. Our study outcome was the change in the initial biologic regimen during the year after the index date. Patients were considered as 'persistent users' if they were still on the index regimen, or 'switchers' if they were on a different biologic at the $365^{\text {th }}$ date of follow-up. We applied 30 days of gap between dispensing after accounting for the days of supply of each dispensing. For sensitivity analysis, we allowed any gap and determined persistent use at $365^{\text {th }}$ date less strictly.

Results: We identified a total of 3,180 TNFi initiators and 214 IL inhibitor initiators (Table). Mean age was $52.9( \pm 11.6)$ years for TNFi initiators and $50.4( \pm 11.7)$ years for IL inhibitor initiators. Using the 30 -day gap, there were $37.1 \%$ persistent TNFi users and $24.8 \%$ persistent IL inhibitor users after 1 year form the index date. $11.1 \%$ of TNFi initiators switched to a different TNFi while $4.7 \%$ switched to an IL inhibitor. Among IL inhibitor initiators, $6.1 \%$ switched to a TNFi and $5.6 \%$ to another IL inhibitors. However, in the sensitivity analysis where we allowed a longer interval between the fills/ injections, there were $53.0 \%$ persistent TNF users and $53.7 \%$ persistent IL inhibitor users. In other words, IL inhibitor patients had a longer interval between the doses than the recommended treatment intervals on the label.

Conclusion: In PsA patients, TNFi initiators were more adherent to the initial regi-

Table. Selected baseline characteristics of initiators TNFi versus IL inhibitors in PsA patients

\begin{tabular}{lcc}
\hline & TNFi & IL inhibitor \\
\hline $\mathrm{N}$ & 3,180 & 214 \\
Age (years), mean (SD) & $52.9(11.6)$ & $50.4(11.7)$ \\
Female, \% & 57.0 & 55.6 \\
Combined comorbidity score, mean (SD) & $0.9(1.8)$ & $0.3(1.2)$ \\
Hypertension, \% & 41.0 & 48.1 \\
Diabetes, \% & 16.4 & 24.8 \\
Psoriasis, \% & 63.2 & 72.9 \\
Non-biologic DMARDs, \% & 66.5 & 60.8 \\
Oral steroids, \% & 49.4 & 41.1 \\
NSAID, \% & 55.4 & 43.0 \\
Emergency room visit, \% & 21.2 & 20.6 \\
Hospitalization & 8.2 & 11.2 \\
No. of visits to dermatologist, mean (SD) & $2.6(8.2)$ & $1.3(4.7)$ \\
\hline
\end{tabular}

men than IL inhibitor initiators during 1-year follow-up period. However, the sensitivity analysis indicates that some patients may resume their initial treatment beyond the indicated refill intervals. Further investigations are needed to clarify whether this is due to a better treatment effectiveness or adverse effects associated with IL inhibitors. Acknowledgments: This study was supported by an investigator-initiated research grant from Pfizer. The sponsor was given the opportunity to make non-binding comments on a draft of the abstract, but the authors retained the right of publication and to determine the final wording.

Disclosure of Interests: Yinzhu Jin: None declared, Sarah Chen Employee of: After finishing the work for this abstract, she has moved to work for Gilead., Hemin Lee: None declared, Joan Landon: None declared, Joseph F. Merola Consultant of: Merck, AbbVie, Dermavant, Eli Lilly, Novartis, Janssen, UCB Pharma Celgene, Sanofi, Regeneron, Arena, Sun Pharma, Biogen, Pfizer, EMD Sorono Avotres and LEO Pharma, Seoyoung Kim Grant/research support from: Seoyoung $\mathrm{C}$ Kim has received research grants from AbbVie, Roche, Bristol-Myers Squibb and Pfizer. DOI: 10.1136/annrheumdis-2020-eular.3721 